\title{
Device-Free Indoor Localization Based on Data Mining Classification Algorithms
}

\author{
Mekuanint Agegnehu Bitew, Rong-Shue Hsiao, ${ }^{*}$ Shinn-Jong Bair, \\ Chiu-Ching Tuan, and Hsin-Piao Lin \\ Department of Electronic Engineering, National Taipei University of Technology, \\ No. 1, Sec. 3, Zhongxiao E. Rd., Taipei 10608, Taiwan, R.O.C.
}

(Received June 2, 2015; accepted November 16, 2015)

Keywords: device-free, indoor localization, statistical classifiers, WiFi interference

Indoor localization is used in many applications such as security, health care, location-based services, and social networking. In traditional localization systems, a target person carries a radio device or sensor and the location of this device is taken as the location of the target person. However, there are situations in which a person does not carry a device. In such cases, devicefree localization (DFL) is the best solution. In this paper, we propose a radio frequency (RF)based DFL system using data mining classification algorithms. ZigBee nodes are deployed at the sides of a rectangular area and the area is divided into square grids. First, a model is developed for each classifier by collecting a received signal strength indicator (RSSI) when a person stands at the center of grids. The RSSI of each RF link is taken as an attribute for classifiers. Second, an online dataset is used to test the trained classifiers. RF links that contribute less for classification are removed from the attribute list. We also analyze the effect of ZigBee and WiFi interference on ZigBee-based DFL systems. Among five data mining classifiers, k-nearest neighbors and support vector machine using sequential minimal optimization achieve a classification accuracy of above $90 \%$.

\section{Introduction}

Recently, indoor localization has shown rapid development with many methods proposed in the literature, most of which consider a target object to carry a device. However, a target object does not always carry a device. Hence, device-free localization (DFL) has been used as a way of detecting and tracking subjects without the need to carry any tag or device. It is suitable for applications such as security, surveillance, assisted living, and elderly care. DFL has the advantage of being unobtrusive while offering good privacy protection.

Researchers have proposed DFL systems using different technologies such as $\mathrm{RF}^{(1-3)}$ camera, ${ }^{(4)}$ infrared, ${ }^{(5)}$ and ultrasonic. ${ }^{(6)}$ Radio frequency (RF)-based schemes have advantages of long range, low cost, and the ability to work through nonconducting walls and obstacles.

RF-based DFL systems are based on the fact that a received signal strength indicator (RSSI) is affected by the presence and movement of people in the monitored environment. ${ }^{(7,8)}$ El-Kafrawy et al. ${ }^{(7)}$ investigated the impacts of human motion on the variances of the RSSI measurement. The

"Corresponding author: e-mail: rshsiao@ntut.edu.tw 
experimental results of Turner et al. ${ }^{(8)}$ indicate that RSSI attenuation varies with the number of people and movement speed.

RF-based DFL systems can be broadly categorized into two: location-based and link-based. Location-based schemes create a radio map with the subject present in various predetermined locations, and then map a test location to one of the trained locations on the basis of observed radio signals. Seifeldin et al. ${ }^{(1)}$ proposed a fingerprinting approach for DFL.

On the other hand, link-based schemes capture the statistical relationship between the RSSI of a radio link and whether the subject is on the line-of-sight (LoS). The subject's location is determined using a geometric approach of the obstructed RF links. A device-free patient localization method proposed by Faraone et al. ${ }^{(2)}$ identifies the obstructed links first and infers the position of the obstacle from a geometric intersection. However, our experimental results indicate that it is difficult to identify obstructed links when longer links are obstructed at the middle.

The accuracy of device-free positioning is highly dependent on the variance of RSSI. A minor environmental change can cause a significant fluctuation in RSSI. Multipath propagation and interference increase variability. RSSI varies with the passage of time even in a static environment.

In this paper, we propose a DFL system based on data mining classification algorithms. RF nodes are deployed at the sides of a rectangular area and the area is divided into square grids. Classification algorithms are trained by collecting RSSI of all links when a person stands at the center of each grid. That is, the RSSI of a link is considered as an attribute and the RSS signature at the center of grids is taken as an instance for the data mining classification algorithms. Links that contribute less for classification are removed from the attribute list to make the classifiers run fast. We compare the performance of five data mining classification algorithms. The effect of ZigBee and WiFi interference on ZigBee-based DFL systems is also discussed.

The rest of the paper is organized as follows. A survey of related works is given in $\S 2$. Section 3 describes our proposed method. Section 4 presents results and discussion, and $\S 5$ concludes the paper.

\section{2. $\quad$ Related Works}

Recently, a number of device-based and device-free localization research studies have been proposed in the literature. Liu et al. ${ }^{(9)}$ presented a survey of wireless indoor positioning techniques. Various measuring principles and algorithms have been presented in this paper.

Among device-based positioning systems, radio frequency identification (RFID)-based tracking and positioning systems are suitable for the care of elderly people because RFID tags are simple to wear. A positioning system integrating RFID with different sensors was proposed by Joshi et al. ${ }^{(10)}$ A big challenge in this system is that elderly people may forget or are unwilling to wear the devices. Hence, DFL is the best option for such type of application.

The variation of the received signal strength due to environmental factors is discussed by $\mathrm{Xu} .{ }^{(11)}$ In this study, he considered the effect of temperature, height of node's position, type of antenna, and the electromagnetic effect of the human body on the RSSI. According to this investigation, a human body at a distance of $5 \mathrm{~m}$ can cause an RSS fluctuation of -3.0 to $3.0 \mathrm{dBm}$.

Xiao and Song proposed a DFL method using outlier link rejection. ${ }^{(3)}$ Owing to the uncertainty of a wireless channel, certain links may be seriously polluted, resulting in error detection. The authors first identified the outlier link and then rejected this link to reduce the error induced by it. 
Han et al. ${ }^{(12)}$ proposed a device-free object tracking system called Twins. Twins leverages the mutual interference between approximated passive tags to enable the motion detection. They discovered an interesting phenomenon: two tags close to each other can enter into a critical state in which one tag is not readable owing to the mutual interference. Any nearby moving objects will inject more RF signals to the twin-pair tags and trigger a change from the unreadable to the readable state. Thus, motion detection is available by observing the state change of twins' tags.

Radio Tomographic Imaging (RTI) technology is used to design a DFL system. ${ }^{(13)}$ However, the performance of RTI is highly dependent on the density of RF nodes. This method will not be feasible if the available RF links are few.

Other proposals use antenna arrays for device-free target detection. ArrayTrack ${ }^{(14)}$ relies on an antenna array and the multiple input multiple output (MIMO) technique to extract the location information from the signal along the direct path (i.e., the first path) and mitigate the multipath effect.

Since both WiFi and ZigBee work on the same frequency band, different techniques have been proposed to address the issue of interference. The performances of ZigBee/IEEE 802.15.4 networks are often evaluated on the basis of the packet reception rate (PRR). ${ }^{(15,16)}$ An adaptive channel selection algorithm has been proposed by Lavric et al. ${ }^{(17)}$ to increase the PRR of ZigBee. ZigBee nodes evaluate the energy level of each ZigBee channel. Then the node will select a channel with the lowest energy level, that is, the least intensity of interference. Zacharias et al. ${ }^{(18)}$ proposed a classification algorithm to detect the common external sources of interference in the 2.4 GHz frequency band.

\section{Proposed Method}

\subsection{Overview of experimental platform}

We deployed 10 XBee series 2 nodes in Room 211 at the complex building of the National Taipei University of Technology, as shown in Fig. 1. The XBee module is attached to an Arduino Fio board that is based on an ATmega328P microcontroller and runs at $3.3 \mathrm{~V}$ and $8 \mathrm{MHz}$ (Fig. 2). RF nodes are deployed at a height of $0.9 \mathrm{~m}$ and the deployment area is divided into $0.5 \times 0.5 \mathrm{~m}^{2}$ grids. All nodes have a capability of transmitting and receiving packets. When one node broadcasts a packet, all other nodes receive and record the RSSI of the link.

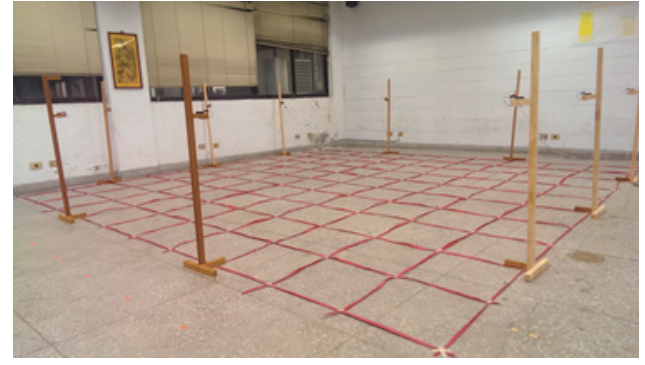

Fig. 1. (Color online) Experimental setup on $5 \times 5$ $\mathrm{m}^{2}$ area.

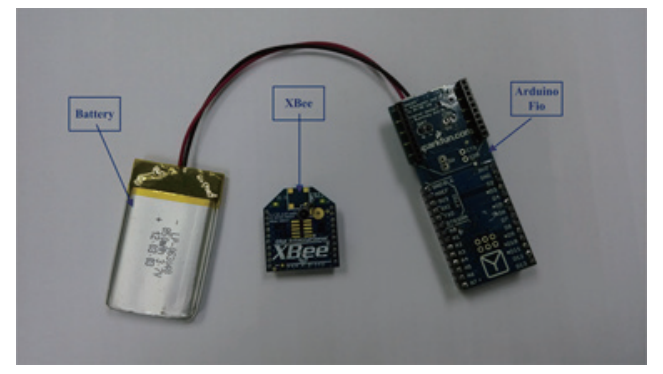

Fig. 2. (Color online) XBee module with Arduino Fio board. 


\subsection{Training and testing classifiers}

Classification is a form of data analysis that extracts models describing important data classes. ${ }^{(19)}$ Data classification is a two-step process, consisting of a training step and a classification step.

In the first step, a classifier is built describing a predetermined set of data classes. This is the training phase, where a classification algorithm builds the classifier by analyzing a training set made up of database tuples and their associated class labels.

An instance, $X$, is represented by an $\mathrm{n}$-dimensional attribute vector, $X=\left(x_{1}, x_{2}, \ldots, x_{n}\right)$, depicting $n$ measurements made on the tuple from $n$ database attributes $A_{1}, A_{2}, \ldots, A_{n}$, respectively. Each instance $X$ is assumed to belong to a predetermined class.

In the second step, the trained classification algorithm is used to classify a new dataset. The accuracy of a classifier on a given test data is the percentage of test set tuples that are correctly classified by the classifier.

Similar to data classification, our localization system has two phases: training and testing. The first phase is training classification algorithms using an offline dataset. When a person stands at the center of a square grid, all XBee nodes broadcast using a token ring algorithm. Nodes that receive a broadcast from a particular node record RSSI. In one round, every node broadcasts once and hence we can obtain the RSS value for all the links in the network. After the end of each round, the RSS signature of a square grid will have the form $\left(R S S_{1}, R S S_{2}, \ldots, R S S_{n}\right)$, where $R S S_{i}$ is the RSS value from link $i$ and $n$ is the total number of links. Hence, the RSS signature is taken as an instance, and the center of the grid where a person stands will be considered as a class for testing and training data mining classifiers. At the center of each grid, 10 RSS vectors are collected (i.e., every class has 10 instances).

For training and testing datasets, RSS from link $i$ is taken as attribute $A_{i}$ and the center of grid $j$ is considered as class $j$ (Table 1). The value of an attribute $A_{i}$ is given in $\mathrm{dBm}$ and $K$ is the number of square grids. In a $5 \times 7 \mathrm{~m}^{2}$ area, both training and testing datasets have 117 classes and 1170 instances (10 instances for each class). WEKA version 3.7 software is used to train and classify datasets.

\subsection{Classification algorithms}

In this section, we will present a detailed description for five data mining classification algorithms: $k$-nearest neighbors $(k-\mathrm{NN})$, support vector machine (SVM) using sequential minimal optimization (SMO), naïve Bayes (NB), naïve Bayes multinomial (NBM), and logistic regression $(\log R)$.

Table 1

Data structure of training and testing datasets.

\begin{tabular}{ccccc}
\hline$A_{1}$ & $A_{2}$ & $\ldots$ & $A_{n}$ & Class \\
\hline-52 & -46 & $\ldots$ & -56 & 1 \\
-53 & -47 & $\ldots$ & -56 & 1 \\
$\ldots$ & & $\ldots$ & $\ldots$ & $\ldots$ \\
-52 & -47 & $\ldots$ & -56 & 1 \\
-44 & -48 & $\ldots$ & -56 & 2 \\
$\ldots$ & $\ldots$ & $\ldots$ & $\ldots$ & $\ldots$ \\
$\ldots$ & $\ldots$ & $\ldots$ & $\ldots$ & $\ldots$ \\
-41 & -49 & $\ldots$ & -55 & $K$ \\
\hline
\end{tabular}




\subsection{1 $k$-NN}

$k$-NN is a powerful and simple method of classification that has proved successful in many applications such as medicine, face recognition, and signature recognition. ${ }^{(19)}$ It is a type of instance-based learning, or lazy learning where the function is only approximated locally. Given a set of training tuples (instances), SVM, NB, and $\operatorname{LogR}$ will construct a generalization model before receiving a test tuple. However, $k$-NN waits until the last minute before doing any model construction to classify a given test tuple. It simply stores a set of training tuples and waits until a test tuple is given. When it sees the test tuple, it classifies the tuple based on its similarity to the stored training tuples.

The steps of $k$-NN algorithm are as follows:

1. Find the Euclidean distance between the test sample $X$ and the previous sample patterns. The

Euclidean distance between two samples $X_{1}=\left(x_{11}, x_{12}, \ldots, x_{1 n}\right)$ and $X_{2}=\left(x_{21}, x_{22}, \ldots, x_{2 n}\right)$ is given by

$$
\operatorname{dist}\left(X_{1}, X_{2}\right)=\sqrt{\sum_{i=1}^{n}\left(x_{1 i}-x_{2 i}\right)^{2}}
$$

2. Select $k$-nearest patterns of the test sample and arrange in increasing order of Euclidean distance.

3. Decide a class for $X$ by a majority vote from $k$-nearest neighbors.

\subsubsection{SVM}

SVM is a data mining classification algorithm for both linear and nonlinear data. ${ }^{(19)}$ It uses nonlinear mapping to transform the original training data into a higher dimension. Within this dimension, it searches for the linear optimal separating hyperplane. The hyperplane is a decision boundary separating the tuples of one class from another.

If the original data is nonlinear, kernel functions are used to project points up in a higher dimensional space hoping that the separability of the data would improve. Determining the best kernel for a particular data set will result in a significant improvement of the performance of SVM. In this paper, we use SVM using the kernel SMO proposed by Platt. ${ }^{(20)}$

\subsubsection{NB}

NB classifiers are statistical classifiers based on the Bayes theorem given by the formula

$$
P\left(C_{i} \mid X\right)=\frac{P\left(X \mid C_{i}\right) P\left(C_{i}\right)}{P(X)}
$$

where $C_{i}$ indicates a class $i, X=\left(x_{1}, x_{2}, \ldots, x_{n}\right)$ is a data tuple considered as evidence, $P\left(C_{i} \mid X\right)$ is a posterior probability of $C_{i}$ conditioned on $X, P\left(C_{i}\right)$ is the prior probability of $C_{i}$, and $P\left(X \mid C_{i}\right)$ and $P(X)$ are the posterior and prior probabilities of $X$, respectively. ${ }^{(19)}$

According to the NB classifier, a data tuple $X$ belongs to a class $C_{\mathrm{i}}$ that has the highest posterior probability conditioned on $X$. That is, $X$ belongs to a class $C_{i}$ if and only if $P\left(C_{i} \mid X\right) \geq P\left(C_{j} \mid X\right)$, for 1 $\leq j \leq m, i \neq j$, and $m$ is the number of classes. 
If the data set contains many attributes, it would be extremely computationally expensive to compute $P\left(X \mid C_{i}\right)$. To reduce the computational cost, the naïve Bayes classifier assumes that the attribute values are conditionally independent of one another. Hence, posterior probability of $X$ given $C_{i}$ is evaluated using

$$
P\left(X \mid C_{i}\right)=\prod_{k=1}^{n} P\left(X_{k} \mid C_{i}\right) .
$$

\subsubsection{NBM}

NBM is a particular type of NB classifier. NB assumes that all of the attributes $\left[x_{k}\right.$ in Eq. (3)] are conditionally independent of one another given some class $C_{i}$. However, the assumption of independency is not always true. To address this issue, NBM assumes each $P\left(x_{k} \mid C_{i}\right)$ in Eq. (3) as multinomial distributions.

\subsection{5 $\log R$}

LogR assumes a parametric form for the distribution $P\left(C_{i} \mid X\right)$, then directly estimates its parameters from the training data. ${ }^{(21)}$ The parametric model assumed by $\operatorname{LogR}$ is given as

$$
P\left(C_{i} \mid X\right)=\frac{\exp \left(w_{i 0}+\sum_{k=1}^{n} w_{i k} x_{k}\right)}{1+\sum_{j=1}^{m-1} \exp \left(w_{j 0}+\sum_{k=1}^{n} w_{j k} x_{k}\right)},
$$

where $i=1,2, \ldots, m-1$.

When $i=m$,

$$
P\left(C_{m} \mid X\right)=\frac{1}{1+\sum_{j=1}^{m-1} \exp \left(w_{j 0}+\sum_{k=1}^{n} w_{j k} x_{k}\right)},
$$

where $w_{j k}$ denotes the weight associated with the $j$ th class $C_{j}$ and with input $X_{k}$. Hence, we can classify any observed RSSI $\mathrm{X}$ to the class $C_{i}$ that maximizes $P\left(C_{i} \mid X\right)$.

\subsection{ZigBee and WiFi interference}

ZigBee is a specification for a wireless standard based on IEEE 802.15.4. IEEE 802.15.4 standards are known to be of low cost, low power, and low data rate. ZigBee uses the unlicensed $2.4 \mathrm{GHz}$ industrial, scientific, and medical (ISM) frequency band. Owing to the scarce availability of the RF spectrum, many technologies are forced to use this unlicensed frequency band. WiFi (IEEE 802.11), ZigBee (IEEE 802.15.4), Bluetooth (IEEE 802.15.1), and cordless phones all share the $2.4 \mathrm{GHz}$ ISM band. In addition, microwave ovens may radiate electromagnetic radiation in the 2.4 GHz band outside the cooking chamber due to imperfect shielding. Hence, a big challenge for ZigBee-based systems is interference with these technologies.

Nowadays, WiFi networks are everywhere in office buildings, homes, and outdoors in urban areas. WiFi and ZigBee RF channels are shown in Fig. 3. In Taiwan, among the 11 ZigBee channels, only two channels (25 and 26) do not overlap with WiFi. In other countries, such as in Europe and Japan, these two channels also overlap with channel 13 of WiFi. 


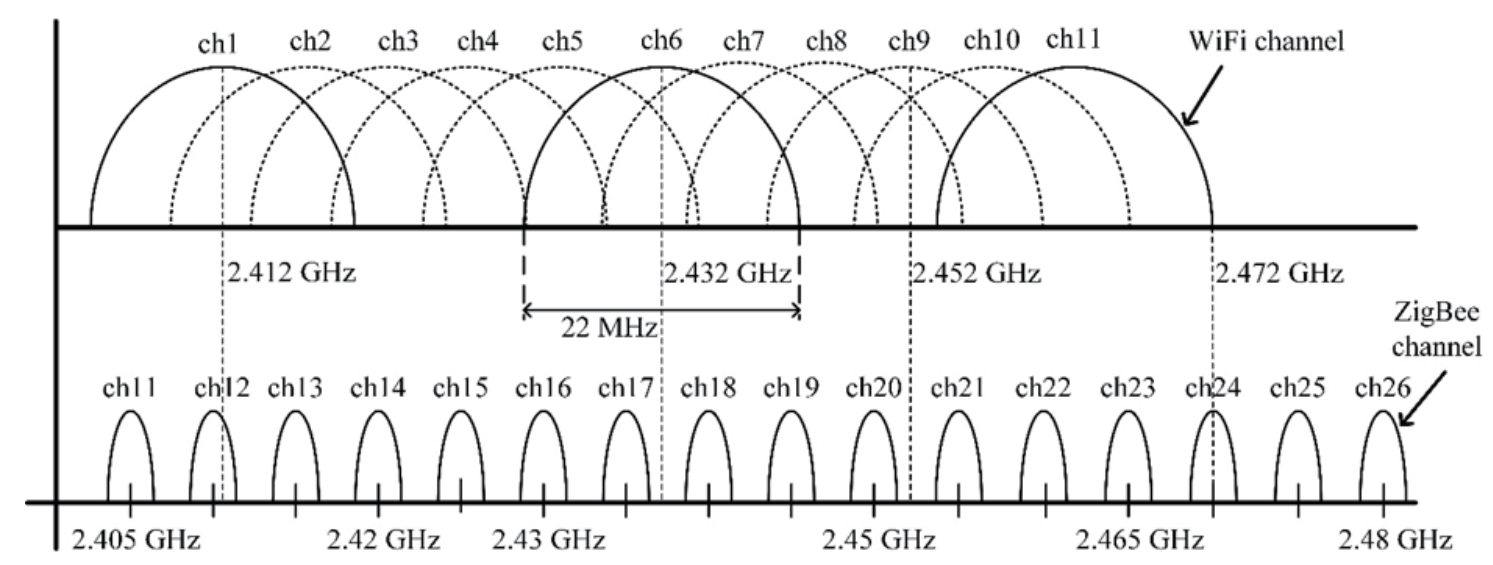

Fig. 3. WiFi and ZigBee channels at $2.4 \mathrm{GHz}$ ISM band.

Since RF channels in ZigBee and WiFi overlap and the transmission power of WiFi transmitters is much higher than those of ZigBee nodes, WiFi highly affects ZigBee networks. The effect of ZigBee and WiFi interference can decrease ZigBee's PRR ${ }^{(15,16)}$ and the variance of RSSI values, and may even stop the functioning of the ZigBee network.

We analyzed the effect of WiFi interference on RSSI values. Experiments were carried out on two ZigBee channels: channel 23 (which overlaps with WiFi channel 11) and channel 26 (nonoverlap). We used a spectrum analyzer to check RF transmissions at the time of the experiment. From our observation, ZigBee channel 26 is free from other wireless transmissions but there were interferences between ZigBee channel 23 and WiFi channel 11. Results indicate that WiFi interference causes an average RSSI variance of $-8 \mathrm{dBm}$. This value is close to the RSSI decrease owing to the presence of a human body on the LoS in ZigBee channels.

\section{Results and Discussion}

We conducted experiments in three areas of 35,25 , and $20 \mathrm{~m}^{2}$. A total of $10 \mathrm{RF}$ nodes are deployed for 35 and $25 \mathrm{~m}^{2}$ areas while eight RF nodes are used for the $20 \mathrm{~m}^{2}$ area. The statistical classifiers $k$-NN, SVM, $\operatorname{LogR}, \mathrm{NBM}$, and NB have been applied to three datasets collected from three experiment areas. We use classification accuracy to compare the performance of these classifiers.

\subsection{RSSI variance with human presence}

We analyzed the effect of a human body on two links of 3 and $7 \mathrm{~m}$ lengths, as shown in Fig. 4. The dots in Fig. 4 indicate the test points. A person will stand at these points at the time of RF transmission.

For the 3-m link, RSSI is measured at 25 test points, among which five test points are on the LoS. When a person is on the LoS, RSSI decreases with at least $-12 \mathrm{dBm}$, as shown in Fig. 5(a). Hence, obstructed links can easily be identified and geometric-based localization methods can be applied. 


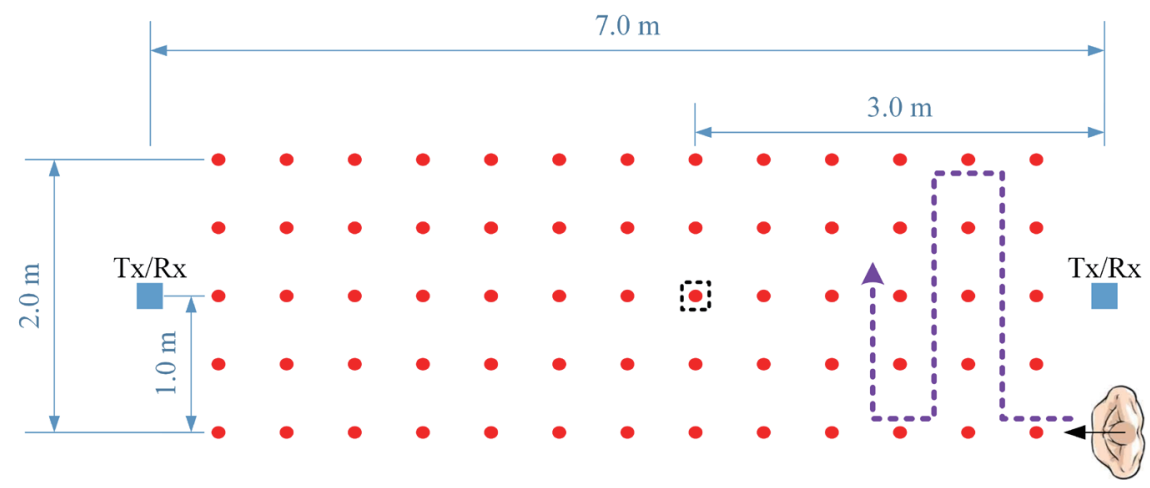

Fig. 4. (Color online) Testing setup for 3-m and 7-m RF links.

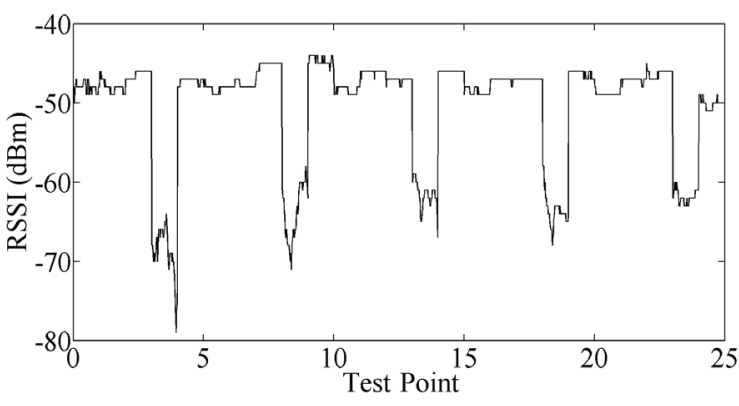

(a)

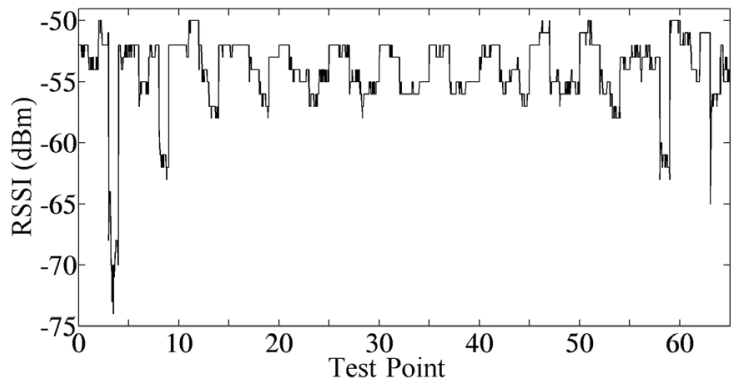

(b)

Fig. 5. RSSI variance. (a) Transmitter and receiver separated by $3 \mathrm{~m}$ and (b) transmitter and receiver separated by $7 \mathrm{~m}$.

For the 7-m link, there are 65 test points between the transmitter and the receiver. Among these points, 13 points are on the LoS. When a test point is on the LoS and closer to the transmitter or receiver (test points: 3, 8, 58, and 63), the decrease in RSSI is very clear [Fig. 5(b)]. For the other nine test points, which are on the LoS but at a distance of more than $2 \mathrm{~m}$ from the transmitter or receiver, the RSSI decrease due to human presence is very small and it is difficult to identify whether a link is obstructed. Hence, the RSSI variance due to human presence in the middle of longer links is very small and this induces localization error in particular for geometric-based methods.

\subsection{Performance of classification algorithms}

Table 2 indicates the performance of five classification algorithms on three experiment areas. The values in the table indicate the percentage of correctly classified instances by each classifier. When the experiment area increases from 25 to $35 \mathrm{~m}^{2}$, keeping the number of RF nodes at 10 , the performances of all the classifiers decrease, because increasing the experiment area decreases the density of links and also the links become longer and less significant.

From the three areas, all the classifiers perform weakly in the $20 \mathrm{~m}^{2}$ area with eight RF nodes (Table 2). This is because the total number of RF links in this area is 28 , which is very few compared with 45 links for the 25 and $35 \mathrm{~m}^{2}$ areas. 
Table 2

Accuracy of classification algorithms for the datasets from 20, 25, and $35 \mathrm{~m}^{2}$ areas.

\begin{tabular}{ccccccc}
\hline Experiment & No. of RF & \multicolumn{5}{c}{ Classification algorithm } \\
\cline { 3 - 6 } area $\left(\mathrm{m}^{2}\right)$ & nodes & $k$-NN & SVM & LogR & NBM & NB \\
\hline 25 & 10 & 90.5 & 90.6 & 88.5 & 84.9 & 78 \\
35 & 10 & 89.6 & 89.4 & 83.8 & 84 & 77 \\
20 & 8 & 82.8 & 81.2 & 72 & 79.5 & 40 \\
\hline
\end{tabular}

Table 3

Effect of WiFi interference on ZigBee-based localization.

\begin{tabular}{ccccccc}
\hline ZigBee & No. of RF & \multicolumn{5}{c}{ Classification algorithm } \\
\cline { 3 - 7 } channel & nodes & $k$-NN & SVM & LogR & NBM & NB \\
\hline Channel 26 & 10 & 90.5 & 90.6 & 88.5 & 84.9 & 78 \\
Channel 23 & 10 & 64.8 & 61 & 60 & 66 & 52 \\
\hline
\end{tabular}

Among the five data mining classifiers, $k$-NN and SVM perform better in all three areas, as shown in Table 2. NB provides the least classification accuracy. NB classifies correctly only $40 \%$ of the instances collected from the $20 \mathrm{~m}^{2}$ area.

\subsection{Effect of WiFi interference}

We conducted experiments on two ZigBee channels (channels 23 and 26) to analyze the effect of WiFi and ZigBee interference on ZigBee-based localization methods. Channel 26 is free from WiFi interference but WiFi channel 11 transmission overlaps with that of ZigBee channel 23 . Comparison of the two channels is carried out in a $5 \times 5 \mathrm{~m}^{2}$ area.

The performances of all the classifiers in ZigBee channel 23 are decreased significantly because ZigBee interferes with WiFi, as shown in Table 3. WiFi interference causes RSSI variance and this will widen the gap between the training and testing datasets. However, if there is no interference, RSSI varies less and the training and testing datasets have the chance to look similar. In ZigBee channel $23, k$-NN classifies $64.8 \%$ of the instants correctly, which is decreased by nearly $26 \%$ from its performance in channel 26. In addition to the decrease in the performance of classifiers, we have observed that ZigBee network stops functioning during the experiment time on channel 23 owing to WiFi and ZigBee interference.

\subsection{Performance of fingerprinting}

We compared our proposed method with fingerprinting. Fingerprinting for device-free localization has been widely used in the literature.(1) For this experiment, 10 RF nodes are deployed in a $25 \mathrm{~m}^{2}$ area. A passive radio map is constructed from 81 training points. For each training point, the RSS signature from 45 links is recorded. The average value of 10 RSSI records is taken for each link. The fingerprinting technique is implemented in ZigBee channels 23 and 26.

Figure 6 indicates the cumulative distribution function (CDF) of localization error. The $1.5 \mathrm{~m}$ accuracy of fingerprinting on channel 26 is $90 \%$. However, the $0.5 \mathrm{~m}$ (grid size) accuracy of $k$-NN and SVM classifiers is more than $90 \%$ on ZigBee channel 26, as shown in Table 3. Similarly, the 1 


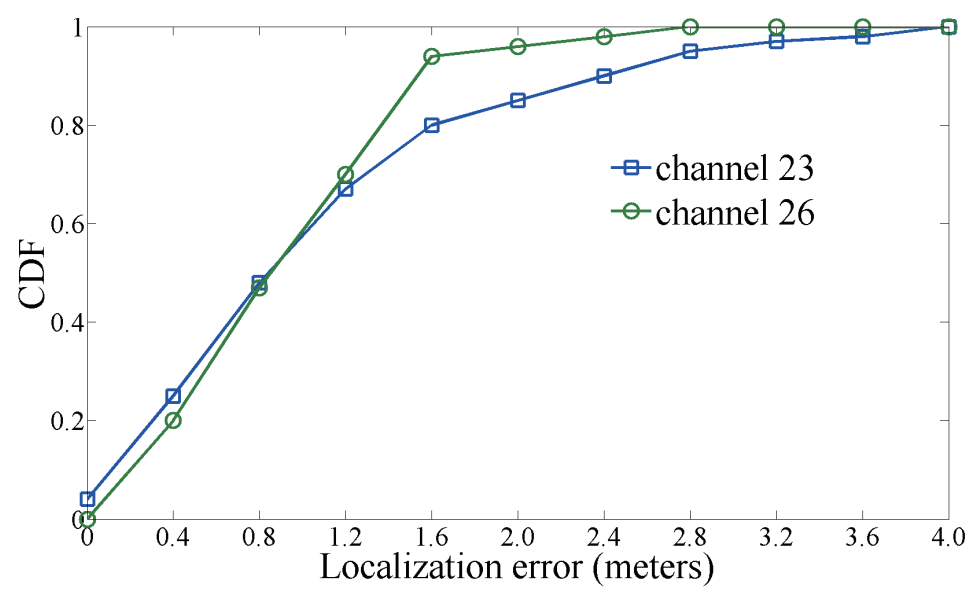

Fig. 6. (Color online) Cumulative distribution function of localization error using fingerprinting.

$\mathrm{m}$ accuracy of fingerprinting on channel 23 is $60 \%$, while the $0.5 \mathrm{~m}$ accuracy for all the classifiers except NB is above $60 \%$, as shown in Table 3 .

\section{Conclusions and Future Works}

In this paper, we have proposed a simple and efficient DFL system based on data mining classifiers. First, a model is developed for each classifier by collecting RSSI from the center of each square grid. RF links that contribute less for classification are removed from the attribute list. This is carried out on the basis of the rank given by WEKA software. In the second phase, a different dataset is used and classifies the test points depending on the developed model.

Experimental results indicate that the RSSI value from longer RF links and those on the edges of the deployment area contribute less for classification. Dense deployment of RF nodes with a link length of less than $6 \mathrm{~m}$ is required to achieve a good localization accuracy. Among the five statistical classifiers, $k$-NN and SVM perform almost the same and better than other classifiers.

We also analyzed the effect of ZigBee and WiFi interference on ZigBee-based DFL. Even though the performances of all the classifiers decreased owing to interference, the localization accuracy is still much better than that of the widely used fingerprinting method.

In future works, the RF node deployment strategy will be explored in detail because the accuracy of DFL is highly dependent on the density of RF node deployment. Also, multichannelbased localization will be explored since several transmissions at different frequency channels increase the available information for location estimation.

\section{References}

1 M. Seifeldin, A. Saeed, A. E. Kosba, A. E. Keyi, and M. Youssef: IEEE Trans. Mobile Comput. 12 (2013) 1321.

2 M. Faraone, R. Alesii, S. Tennina, and F. Graziosi: Proc. IEEE Int. Workshop Comput. Aided Model. Des. Commun. Links Netw. (IEEE, Athens, 2014) pp. 149-153.

3 W. Xiao and B. Song: Math. Prob. Eng. 2015 (2015) 8. 
4 J. M. Sanchez-Matamoros, J. R. M.-d. Dios, and A. Ollero: Proc. IEEE Int. Conf. Mechatron. (IEEE, Malaga, 2009) pp. 1-9.

5 S. Lee, K. N. Ha, and K. C. Lee: IEEE Trans. Consum. Electron. 52 (2006) 1311.

6 T. W. Hnat, E. Griffiths, R. Dawson, and K. Whitehouse: Proc. ACM Conf. Embedded Netw. Sens. Syst. (ACM, Toronto, 2012) pp. 309-322.

7 K. El-Kafrawy, M. Youssef, and A. El-Keyi: Proc. IEEE Int. Symp. Pers. Indoor Mobile Radio Commun. (IEEE, Toronto, 2011) pp. 1208-1212.

8 J. S. C. Turner, M. F. Ramli, L. M. Kamarudin, A. Zakaria, A. Y. M. Shakaff, D. L. Ndzi, C. M. Nor, N. Hassan, and S. M. Mamduh: Proc. IEEE Conf. Wireless Sens. (IEEE, Kuching, 2013) pp. 30-35.

9 H. Liu, P. Banerjee, and L. Liu: IEEE Trans. Syst., Man Cybern. Part C Appl. Rev. 37 (2007) 1067.

10 G. P. Joshi, S. Acharya, C. S. Kim, B. S. Kim, and S. W. Kim: Int. J. Dist. Sens. Netw. 2014 (2014) 11.

11 L. Xu: Proc. Int. Conf. Adv. Comput. Control (IEEE, Harbin, 2011) pp. 151-154.

12 J. Han, C. Qian, X. Wang, D. Ma, J. Zhao, P. Zhang, W. Xi, and Z. Jiang: Proc. IEEE Int. Conf. Comput. Commun. (IEEE, Toronto, 2014) pp. 469-476.

13 J. Wilson and N. Patwari: IEEE Trans. Mobile Comput. 11 (2012) 947.

14 J. Xiong and K. Jamieson: Proc. USENIX Conf. Netw. Syst. Des. Implementation (USENIX, Lombard, 2013) pp. $71-84$.

15 Y. Tang, Z. Wang, T. Du, D. Makrakis, and H. Moutftah: Proc. Consumer Commun. Netw. Conf. (IEEE, Las Vegas, 2013) pp.765-768.

16 W. Yuan, X. Wang, J. P. Linnartz, and L. J. Niemegeers: Wireless Pers. Commun. 68 (2013) 281.

17 A. Lavric, V. Popa, and S. Sfichi: Elektronika IR Elektrotechnika 19 (2013) 105.

18 S. Zacharias, T. Newe, S. O’Keeffe, and E. Lewis: Int. J. Distrib. Sens. Netw. 2015 (2015) 24.

19 J. Han and M. Kamber: Data Mining Concepts and Techniques (Morgan Kaufmann, San Francisco, 2006).

20 J. Platt: Microsoft Research Technical Report MSR-TR-98-14 (1998).

21 T. M. Mitchell: Machine Learning (McGraw Hill, New York, 1997). 